\title{
Lifetime measurements of excited nuclear states of astrophysical interest via the Doppler Shift Attenuation Method
}

\section{Clemens Herlitzius ${ }^{1}$}

Physik Department E12, Technische Universität München

James Franck Strasse, 85748 Garching, Germany

E-mail: clemens.herlitzius@ph.tum.de

\section{Shawn Bishop}

Physik Department E12, Technische Universität München James Franck Strasse, 85748 Garching, Germany

E-mail: shawn.bishop@ph.tum.de

\section{Janina Fiehl}

Physik Department E12, Technische Universität München

James Franck Strasse, 85748 Garching, Germany

E-mail: janina.fiehl@ph.tum.de

The Doppler Shift Attenuation Method (DSAM) is a known technique to measure lifetimes of excited states in the range of fs up to ps. The energy of a Doppler shifted $\gamma$-ray, which is emitted by a decelerating de-exciting nucleus, is measured with a HPGe detector. The lifetime of the excited state can then be extracted from the Doppler shifted $\gamma$-ray energy spectrum.

\begin{abstract}
A DSAM facility to measure lifetimes of nuclei of astrophysical interest has been built by the nuclear astrophysics group at the Technische Universität München. First tests and experiments have been done in the end of August this year at the Maier-Leibnitz-Laboratorium in Munich. A study of a ${ }^{32} \mathrm{~S}$ beam into a ${ }^{3} \mathrm{He}$ implanted gold target has been used to test and understand the detector system. Experiments to study lifetimes of astrophysical interest will be performed in the future.
\end{abstract}

11th Symposium on Nuclei in the Cosmos - NIC XI

Heidelberg, Germany

July 19-23 2010

\footnotetext{
$1 \quad$ Speaker
} 


\section{Classical Nova Explosions}

A classical nova explosion requires a binary star system comprised of a white dwarf (WD) and a main sequence or red giant companion. In these systems hydrogen-rich material is transferred from the companion onto the white dwarf. The hydrogen accumulates on the surface of the WD, creating a hot, dense envelope of H-rich material. Because of its small radius (a few thousands of $\mathrm{km}$ ) and a mass of $\sim 1.4 \mathrm{M}_{\odot}$ (less than the Chandrasekhar mass), the material at the base of the envelope is degenerate [1].

Energy is rapidly produced, when the temperature and the density become high enough to start the pp chain fusion and the $\mathrm{CNO}$ cycle, and due to the degenerate conditions, no expansion, and therefore no cooling, of the envelope occurs. This provides for a situation where the energy production rate can grow faster than its transport out of the envelope.

This mechanism leads to a thermonuclear runaway, which occurs on the white dwarf's surface. The WD itself is not destroyed by the ejection of its surface. About $10^{-4}-10^{-5} \mathrm{M}_{\odot}$ are ejected during a nova event for around $\mathrm{M}_{\mathrm{wd}}<1.44 \mathrm{M}_{\odot}$. The explosion can generate a luminosity increase of a factor of $10^{4}-10^{6}$ which returns to normal after 10-250 days. The binary star system survives the ejection and the process of transferring material is repeated. The period of the whole process can be $10^{4}-10^{5}$ years $[1,2]$.

\subsection{Resonant Nuclear Reaction Rates}

Energy production and subsequent nucleosynthesis in a nova explosions are determined by the rates of $(\mathrm{p}, \gamma)$ reactions leading up to, and during the thermonuclear runaway. These reaction rates are dependent on the temperature, densities and - since resonant nuclear reactions play a key role - the properties of excited nuclear states the compound nuclei formed by $(\mathrm{p}, \gamma)$.

Nuclear network calculations are used to understand the processes and to test theoretical models of the explosion mechanism and the abundances produced. These methods require the $(p, \gamma)$ reaction rate. Reaction rate sensitivity studies, where reaction rates in network calculations are varied, show, that the final abundances produced in nova events are influenced by presently unknown resonant $(\mathrm{p}, \gamma)$ reaction rates [3]. The resonant reaction rate is given by the energy integral over the product of the relative energy of the entrance channel, the $(p, \gamma)$ cross section and the Boltzmann distribution factor [4]. Since we look to narrow resonances, the cross section is represented by the Breit-Wigner expression with narrow total width. With this assumption, the integral can be analytically calculated and becomes a sum over all contributing resonances in the Gamow window; namely,

$$
\langle\sigma v\rangle=\left(\frac{2 \pi}{\mu k T}\right)^{3 / 2} \hbar^{2} \sum_{i} \omega \gamma_{i} e^{-\frac{E_{i}}{k T}}
$$

where $\langle\sigma v\rangle$ is the reaction rate, $\mu$ is the reduced mass, $\mathrm{k}$ is the Boltzmann factor, $\mathrm{T}$ is the temperature and $\mathrm{E}_{\mathrm{i}}$ is the resonance energy. $\omega \gamma$ is the resonance strength, defined by

$$
\omega \gamma_{i}=\frac{2 J_{i}+1}{\left(2 J_{p}+1\right)\left(2 J_{X}+1\right)} \frac{\Gamma_{p} \Gamma_{\gamma}}{\Gamma_{p}+\Gamma_{\gamma}}=g\left(1-B_{p}\right) B_{p} \frac{\hbar}{\tau}
$$


Where $\mathrm{J}_{\mathrm{i} / \mathrm{p} / \mathrm{X}}$ are the spins of the resonance state/projectile/target, $\Gamma_{\mathrm{p} / \gamma}$ are the partial widths of the $\mathrm{p} / \gamma$ decay, $\mathrm{B}_{\mathrm{p}}=\Gamma_{\mathrm{p}} / \Gamma$ is the proton branching ratio and $\tau$ is the lifetime of the state.

From the final expression, we see that the resonant rate is inversely dependent on the lifetime of the nuclear state into which the proton captures. This quantity can be measured with the Doppler shift attenuation method.

\section{The Doppler Shift Attenuation Method}

Lifetimes of excited states in the range of $10^{-14}-10^{-11} \mathrm{~s}$ have been measured with the Doppler shift attenuation method [5]. The excited nucleus of interest is produced and populated via a direct reaction mechanism in a setup where the beam is completely stopped in the target. The target functions as a reactant and also as the stopping material for the excited nucleus of interest. This implies the use of an implanted target. The nuclear reactions of interest take place at the entrance of the target and the produced excited nucleus is decelerated and stopped within the thickness of the target foil. Typical stopping materials are gold or other high- $\mathrm{Z}$ metals, where implantation is possible. The timescale of the deceleration until stopping and the lifetime of the excited states must be comparable. When de-excitation via $\gamma$ decay is the preferred channel, the lifetime can be extracted from the spectrum of Doppler shifted $\gamma$ ray. Depending on the velocity at the moment of de-excitation, the energy of the emitted $\gamma$-ray is Doppler shifted. When $\beta<c$ c, the velocity of the excited nucleus $D^{*}$ at its moment of decay is given by,

$$
A+b \longrightarrow c+D^{*} \text {. }
$$

Where $\mathrm{A}$ is the beam particle, $\mathrm{b}$ is the reactant in the target, $\mathrm{c}$ is the light eject product and D* is the excited nucleus of interest.

$$
\beta_{0} F(\tau) \cos \Phi=\left(\frac{E_{\gamma}}{E_{0}}-1\right)
$$

Where $\mathrm{E}_{\gamma}$ is the measured $\gamma$-ray energy, $\mathrm{E}_{0}$ is the non-shifted g-ray energy of the deexcitation, $\beta_{0}$ is the velocity of $\mathrm{D}^{*}$ directly after the reaction, $\phi$ is the $\gamma$-ray angle with respect to the initial direction of $\mathrm{D}^{*}$ and $\mathrm{F}(\tau)$ is the Doppler attenuation factor.

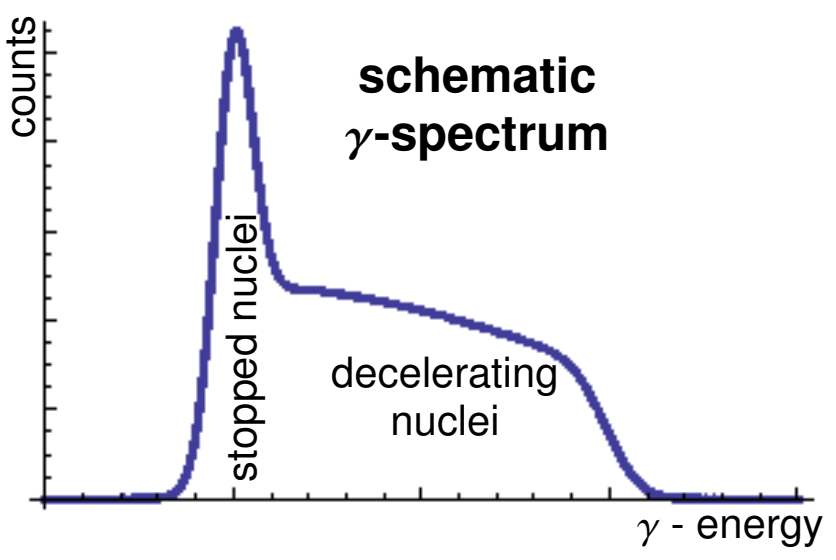

Figure 1: The left schematic spectrum demonstrates the measured energy for one specific de-excitation energy with Doppler shift. The peak represents events, where the nuclei have been stopped before decay. Events with higher energy represent nuclei, which decay during the deceleration and therefore underlie the Doppler shift. 
The energy and exit angle of the light ejectile "c" is measured by silicon detectors to reconstruct the reaction kinematics and, thereby, specify the populated state. The measured $\gamma$-ray energy spectrum provides the velocity distribution of the nucleus $\mathrm{D}^{*}$ at the moment of its decay. Knowing the stopping power of the target material, one can model the velocity distribution of $D^{*} \beta$ as a function of its decay time. A folding of this velocity distribution with the exponential decay probability gives the Doppler attenuation factor $\mathrm{F}(\tau)$,

$$
F(\tau)=\frac{1}{\tau \beta_{0}} \int_{0}^{\infty} \beta(t) e^{-t / \tau} d t
$$

A fit of the above formula for stopping power function provides the lifetime $\tau$ [6].

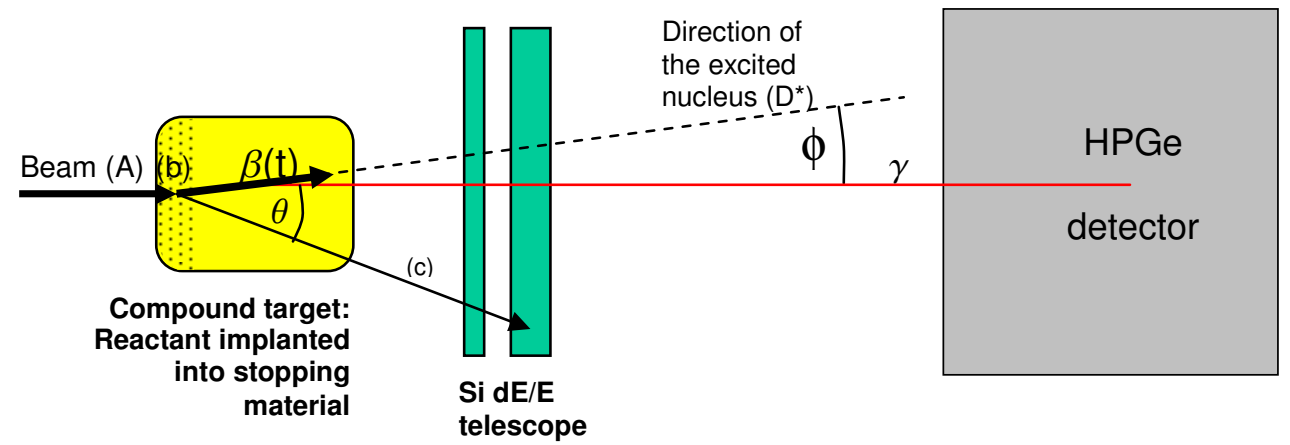

Figure 2: This diagram shows the schematic setup of a Doppler shift station. The incident beam particle "A" reacts with implanted "b" at the target entrance. $\mathrm{D}^{*}$ is populated and is stopped within the target. Because of c's low atomic number, it can penetrate the target and is detected and identified in the $\mathrm{dE} / \mathrm{E}$ Silicon detectors. Due to the two

body reaction, c's exit angle and energy provide information about the reaction channel and excitation of $\mathrm{D}^{*}$.

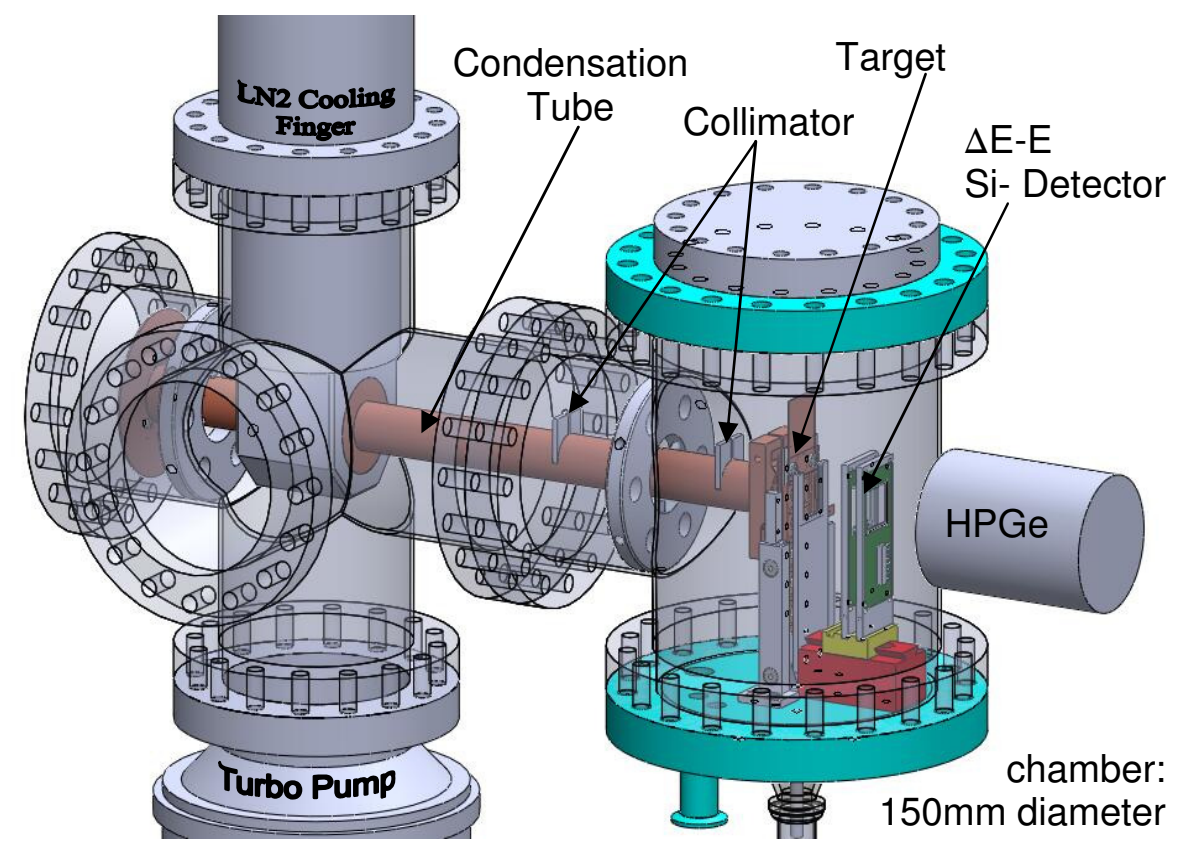

Figure 3: The design diagram of the DSAM facility

The design diagram of the setup shows the condensation tube along the beam axis. The targets can be cooled, when the target ladder is attached to the condensation tube. The target 
stops all heavy ions and only light ejectile particles from the reaction can penetrate the target foil and are detected in the $\Delta$ E-E Silicon detectors. Outside the chamber, a $95 \%$ efficiency, ntype co-axial HPGe detector is used to measure the $\gamma$ energy. The crystal volume is $\sim 375 \mathrm{~cm}^{3}$.

In a first test experiment, an $80 \mathrm{MeV}^{32} \mathrm{~S}$ beam has been used on a ${ }^{3} \mathrm{He}$ implanted gold target. Possible population of energy states in ${ }^{34} \mathrm{Cl},{ }^{33} \mathrm{Cl},{ }^{32} \mathrm{Cl}$ and the measurement of their lifetimes will be used to understand the system and to commission the whole facility.

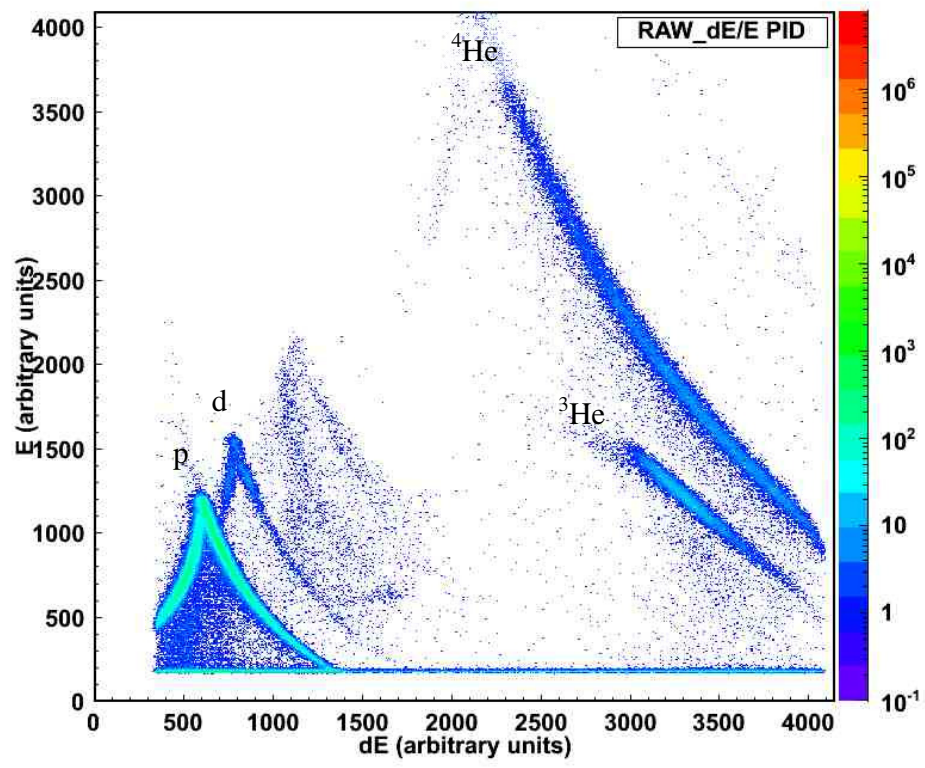

Figure 4: Particle ID plot from the dE/E Silicon telescope, showing clear lines of protons (p), deuterons (d), alphas $\left({ }^{4} \mathrm{He}\right)$ and elastically scattered ${ }^{3} \mathrm{He}$.

The experiment has been performed at the Maier-Leibnitz-Laboratorium of LMU and TUM in Garching and the analysis on the Doppler shifted gamma ray spectrum is proceeding.

This research was supported by the DFG cluster of excellence 'Origin and Structure of the Universe' (www.universe-cluster.de).

\section{References}

[1] C. E. Rolfs / W. S. Rodney, Cauldrons in the Cosmos, The University of Chicago Press 1988

[2] J. José, M. Hernanz, C. Iliadis, Nucleosynthesis in classical novae, Nuclear Physics A 777 (2006) $550-578$

[3] C. Iliadis, J. José, S. Starrfield, P. Tupper, "The Effects of Thermonuclear Reaction-Rate Variations on Nova Nucleosynthesis: A Sensitivity Study", The astrophysical Journal supplement series, 142:105-137 (2002)

[4] D.G. Sargood, "Charged particle reaction cross sections and nucleosynthesis", Phys. Rep. Vol. 93, 2 (1982)

[5] A. E. Blaugrund, "Notes on Doppler-shift lifetime measurements", Nuclear Physics 88 (1966) 501512

[6] E.K. Warburton, J.W. Olness, A.R. Poletti, „Nuclear Structure of Na22.I.Gamma-Ray Correlations and Lifetime Measurements for Levels of Eex <3.1 MeV*”, Phys rev. Vol. 160 Number 4 (1967) 